Saudi Journal of Oral and Dental Research

Abbreviated Key Title: Saudi J Oral Dent Res ISSN 2518-1300 (Print) |ISSN 2518-1297 (Online) Scholars Middle East Publishers, Dubai, United Arab Emirates Journal homepage: http://scholarsmepub.com/sjodr/

\title{
Ameloblastoma Simulating A Dentigerous Cyst: A Case Report
}

\author{
Ouertani Hend ${ }^{1 *}$, Teffeha Ghaida ${ }^{2}$, Jemaa Mayada ${ }^{1}$, Jegham Hela ${ }^{1}$, Khattech Mohamed Bassem ${ }^{1}$
}

${ }^{1}$ Military Hospital of Tunisia, Mont-Fleury 1008 Tunis, Tunisia

${ }^{2}$ Faculty of Dental Medecin of Monastir, Avicenne Avenue 5019, Monastir, Tunisia

\author{
DOI: $\underline{10.36348 / \text { sjodr.2019.v04i11.006 }}$ | Received: 14.11.2019| Accepted: 20.11.2019 | Published: 30.11 .2019 \\ *Corresponding author: Ouertani Hend
}

\section{Abstract}

Ameloblastomas are bening intraosseous lesions affecting maxillary jaws. They originate from the epithelium involved with the formation of teeth such as enamel, odontogenic rests of Malassez, reduced enamel epithelium and odontogenic cyst lining. Thus, the hypothesis of transformation of a dentigerous cyst into an ameloblastoma was suggested in the literature. These lesions are locally invasive and can grow to infiltrate soft tissues. The risk of recurrence after surgical treatment is important and a long term survey is indicated. The aim of this paper is to demonstrate the diagnostic dilemma in front of a well-defined radiolucent lesion of the mandibule associated to an impacted wisdom tooth and the diffuclties on therapeutic decision. A healthy 34-year-old man referred to our dentistry department complaining about the recurrence of inflammatory episodes associated with tooth number 48 . The radiological examination showed a large well limited radiolucent lesion related to tooth number 48 , situated in the ramus region. The patient underwent tooth extraction and cystic enucleation. The anatomopathological examination confirmed the diagnosis of a follicular ameloblastoma. Nevertheless, the cystic wall showed both a detigerous cystic portion in one part and a follicular ameloblastoma segment in another part, confirming the hypothesis of ameloblastoma transformation of a detnigerous cyst.

Keywords: Ameloblastoma - dentigerous cyst - wisdom tooth - surgical treatment.

Copyright @ 2019: This is an open-access article distributed under the terms of the Creative Commons Attribution license which permits unrestricted use, distribution, and reproduction in any medium for non-commercial use (NonCommercial, or CC-BY-NC) provided the original author and source are credited.

\section{INTRODUCTION}

Ameloblastoma is a benign intraosseous lesion affecting maxillary jaws. It originates from the residual epithelium of the tooth germ, epithelial rests of Malassez, epithelium of odontogenic cysts stratified squamous epithelium and epithelium of the enamel organ.

Ameloblastoma is locally invasive, and it can infiltrate soft tissues after growth, the risk of recurrence after surgical treatments is high; thus, a long-term survey is recommended.

In 2017, the classification of the WHO presented different types of ameloblastoma: solid, multicystic, unicystic, and peripheral type [1].

The unicystic type is a variant of ameloblastoma that occurs as a single cystic cavity. It can be associated with an impacted tooth.

In the present article, we report a case of ameloblastoma related to an impacted tooth simulating a dentigerous cyst by detailing the pathogenesis and differential diagnosis and also we will discuss the hypothesis of transformation of a dentigerous cyst into an ameloblastoma.

\section{OBSERVATION}

A healthy 34-year-old man referred to our dentistry department complaining about the recurrence of inflammatory episodes associated with tooth number 48. At clinical examination, we noticed a discreet swelling of the right cheek. The assessment of the trigeminal and facial nerves showed that their functions were intact. No lymphadenopathy was detected Figure1.

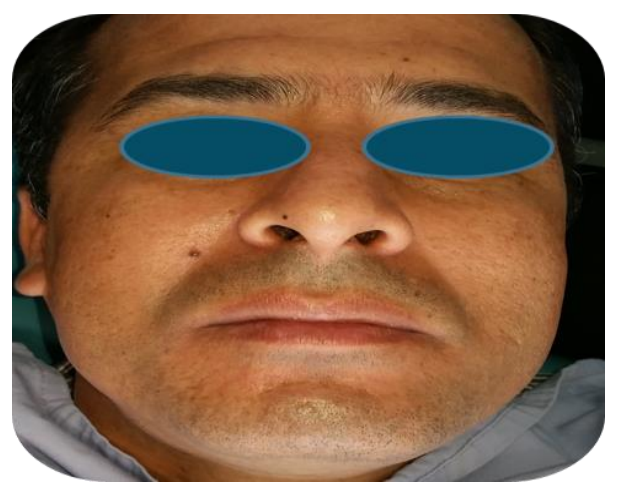

Fig-1: Extrabuccal view of a 34-year old men complaining about the recurrence of inflammatory episodes associated with tooth number 48 , with a discret swelling of the right cheek 
Intraoral examination revealed a partially erupted wisdom tooth number 48 .

The orthopantomogram (OPG) showed a wellcircumscribed, radiolucent lesion in the right posterior mandible, extending to the ramus and associated with the wisdom tooth Figure-2.

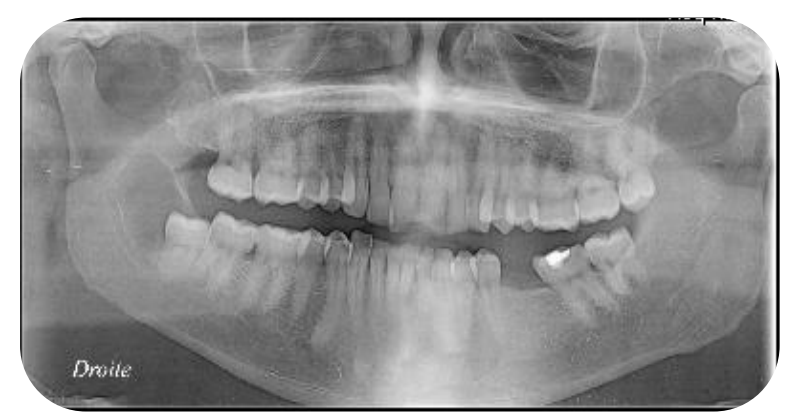

Fig-2: Orthopantomogram of a 34-year old men presenting a well-circumscribed, radiolucent lesion in the right posterior mandible, extending to the ramus and associated with the wisdom tooth

The Cone Beam/Computed Tomography (CBCT) scan revealed a benign expansion of cortical bone eroding the internal and external cortical plate of the ramus.

We noted the continuity of the lesion with the crown of the tooth number 48 and its insertion on the cementoenamel junction (CEJ). The crown of the unerupted wisdom tooth was within the lesion Figures3.

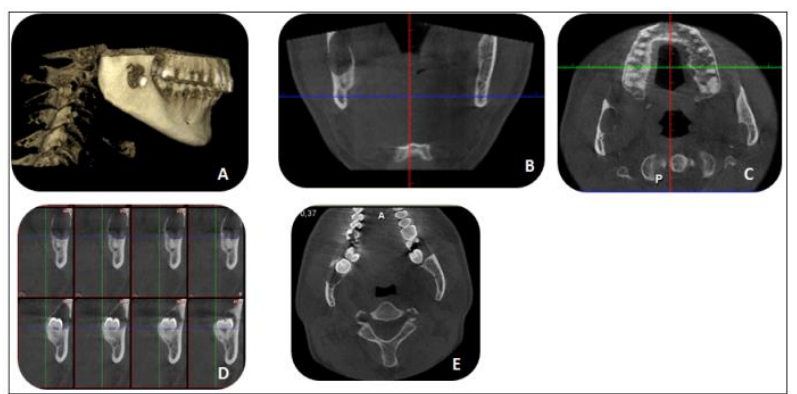

Fig-3: CB/CT scan of a 34-year old men revealing a benign radiolucent lesion with expansion of cortical bone eroding the internal and external cortical plate of the ramus $(A, B, C)$, in continuity with the crown of the tooth number 48 having insertion on the cementoenamel junction. The crown of the unerupted wisdom tooth was within the lesion $(D, E)$

The diagnosis of the dentigerous cyst was retained. Nevertheless, the internal and external cortical disruption of the ramus suggested possible aggressivity of the lesion: a keratocyst or ameloblastoma were also suspected.

The patient underwent tooth extraction with cyst enucleation under local anesthesia with no relevant postoperative complications. On gross examination, we noticed a difference in the texture of the cystic wall: on one side the wall was fine reminiscent of a dentigerous cyst, and on another side, the wall was more like a thickened whitish mass Figures-4.

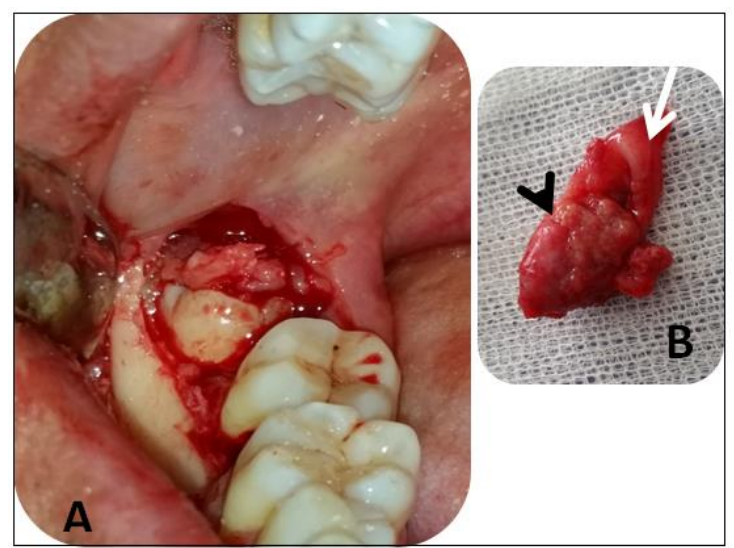

Fig-4: A: Per-operative view of the tooth number 48 in the mandibular lesion, B: On gross examination a difference in the texture of the cystic wall was present: on one side the wall was fine reminiscent of a dentigerous cyst (white arrow), and on another side, the wall was more like a thickened whitish mass (black arrow)

The anatomopathological examination concluded that it was an unikystic ameloblastoma. The cystic portion of the mass contained straw-colored fluid and had a uniform thin non-keratinized epithelium with 2-3 layers of flattened cells. The substantial portion was determined to be the follicular type. Therefore, the hypothesis of the transformation of the dentigerous cyst into ameloblastoma was confirmed.

Follow-Up examinations during the last three years showed that the patient had no signs of recurrence.

\section{DISCUSSION}

Ameloblastoma is the most common benign odontogenic tumor. It is an intraosseous progressively growing epithelial neoplasm, characterized by local expansion and tendency for recurrences [2, 4]. The annual incidence is estimated at 0.5 cases per million population. The age range is 8-92 years with no differential distribution between men and women. The most affected patients' average age is between 40 and 50 years old $[1,4]$.

Ameloblastoma is found generally in the mandible, in the posterior region like described in our case. The anterior mandible is the second frequent localization, followed by the posterior maxilla and anterior maxilla $[1,4]$.

The slowing, painless expansion lesion is generally, diagnosed at a late stage. That is when its volume becomes important, causing facial deformation. Other complications can be revealing: paraesthesia, dental mobility (less frequently), trismus, and difficulty of mastication once soft tissues are invaded $[2,4]$. 
The radiographic examination is primordial for diagnosis. It shows commonly a corticated multilocular osteolytic lesion called soap-bubble [1, 2, 4].

Although, our case displayed a unilocular appearance which is, in fact, less common. Moreover, the association with an unerupted tooth is rarer. Stathopoulos et al., found that $0.27 \%$ of the wisdom teeth were associated with ameloblastoma development [8].

In this situation, we faced a diagnostic dilemma. In fact, after radiographic examination in our case, we were more oriented to a dentigerous cyst.

Indeed, dentigerous cysts in term of frequency, are the most common non-inflammatory odontogenic cysts, developed surrounding an unerupted tooth with an accumulation of fluid between the follicular epithelium and the crown. For larger extended lesions, we can find a multilocular aspect with septa: in those cases, differential diagnosis is more confusing as dentigerous cysts could be confounded with ameloblastomas $[4,5,7]$.

A keratocyst, wich can also be associated with impacted teeth, may be suggested as another differential diagnosis as it has the same radiological aspect [2, 4]. It shows in radiography as a sizeable radiolucent lesion with more aggressive growth with the budding of the basal layer. Sometimes, we notice the existence of daughter cysts outside the primary lesion.

Less commonly, we may suspect an odontogenic tumor (odontogenic myxoma) or nonodontogenic lesions such as traumatic bone cyst appearing as a large osteolytic lesion associated with an impacted tooth [4].

A contrast-enhanced maxillofacial computed tomography is the best radiographic technique to use with bone and soft-tissue window settings. It shows the lesion limits, exteriorization with cortical disruption and internal septations, repression of the mandibular nerve, and sometimes the existence of daughter cysts outside the primary lesion $[4,7]$.

However, in our case, we noted a relatively small size of the lesion and the panoramic X-Ray was evidence of a well-defined aspect. Therefore, we required a $\mathrm{CB} / \mathrm{CT}$ scan.

Perhaps if we performed a CT scan, we could have been able to see the differences in texture of the cystic wall in the soft-tissue window with his cystic and solid components.

The histopathological study is essential for the final diagnosis. There are many types of ameloblastomas based on the histopathological examination. The most common type is the follicular type, described in our case. We found resemblance with the enamel organ within the fibrous stroma. A cylindric to cuboidal (ameloblast-like) cells on the external site with hyperchromatic nuclei arranged in a palisading pattern with reverse polarity in association to stellate reticulum in the central core were found. The conjonctif stroma was dense $[1,6]$.

The second most common type is the plexiform type. Other types, including acanthomatous, granular, and basaloid, can be found.

Ameloblastomas may arise from various sources of odontogenic epithelium, in general from the dental lamina, including the epithelial lining of the dental follicle [1, 4]. The expression of early dental epithelial markers such as PITX2, MSX2, DLX2, RUNX1, and ISL1, confirm the dental lamina origin of ameloblastomas [1, 6, 7, 9]. Approximately 50\% of ameloblastomas arises from neoplastic alteration in the epithelial lining of a dentigerous cyst. This neoplastic change might be local like seen in our case report in the histopathological findings $[2,3,5,6,7]$.

The ideal treatment choice for ameloblastoma is extensive surgical resection with a margin of at least $10 \mathrm{~mm}$ (the resection of the lesion with the daughter lesions generally situated in the peripheral zone) in order to minimize the risk of recidivism $[1,3,6]$.

Generally, the decision for surgical treatment is based on radiological findings.

We have different option treatment to manage small radiolucent images related to an impacted tooth, like in our case. They vary from conservative approaches, including enucleation or marsupialization to more radical treatment procedures with extensive surgical resection once we suspect more aggressive lesions such as ameloblastoma [4, 10, 11].

The optimal treatment choice is critical in cases such presented in this paper. We first made the diagnosis of a dentigerous cyst. Thus, the treatment was based on simple enucleation. Therefore, we take note of the high risk of recurrence. In these cases, the place of longtime follow-up is primordial to detect recidivism $[1,10]$.

In the case of recurrences, the ideal treatment becomes extensive surgical resection, including an area of bone beyond radiographical margins. Especially that, conservative surgery has a high recurrence rate $(60$ $80 \%)[1,2]$.

More than $50 \%$ of recurrences occur within five years after initial treatment. We should do a longtime survey, at least for 25 years; but lifelong follow-up should be considered $[1,2]$. 
In summary, this case report is proof of ameloblastic transformation of dentigerous cysts based on the association of dentigerous cysts with unerupted tooth and ameloblastoma.

These findings rely heavily on histopathologic evaluation, especially that in our observation the histological findings showed the signs of a dentigerous cyst on one side of the cystic wall and the ameloblastoma (follicular type) on another side. of interest.

The authors declare that they have no conflict

Informed consent was obtained from the patient for the material in this paper to be published.

Submission for publication of the manuscript has been approved by all the authors as well as by the institutions where the work had been performed.

\section{REFERENCES}

1. El-Naggar A. K., Chan J. K. C., Grandis. J. R., Takata T., Slootweg P. J. 2017. (Eds): WHO Classification of Head and Neck Tumors (4th Edition). IARC: Lyon.

2. Friedrich, R. E., \& Zustin, J. (2010). Ameloblastoma of the maxillary sinus 11 years after extirpation of extensive dentigerous cysts and dystopic wisdom tooth. in vivo, 24(4), 567-570.

3. Holmlund, A., Anneroth, G., Lundquist, G., \& Nordenram, Å. (1991). Ameloblastomas originating from odontogenic cysts. Journal of oral pathology \& medicine, 20(7), 318-321.

4. Cankurtaran, C. Z., Branstetter IV, B. F., Chiosea, S. I., \& Barnes Jr, E. L. (2010). Ameloblastoma and dentigerous cyst associated with impacted mandibular third molar tooth. Radiographics, 30(5), 1415-1420.

5. Meleti, M., \& Van der Waal, I. (2013). Clinicopathological evaluation of 164 dental follicles and dentigerous cysts with emphasis on the presence of odontogenic epithelium in the connective tissue. The hypothesis of "focal ameloblastoma". Medicina oral, patologia oral y cirugia bucal, 18(1), e60-e64.

6. Gunawardhana, K. S. (2013). Diagnostic dilemma of unicystic ameloblastoma: novel parameters to differentiate unicystic ameloblastoma from common odontogenic cysts. Journal of Investigative and Clinical Dentistry, 5, 1-6.

7. Barrett, A. W., Sneddon, K. J., Tighe, J. V., Gulati, A., Newman, L., Collyer, J., ... \& Liebmann, R. D. (2017). Dentigerous Cyst and Ameloblastoma of the Jaws: Correlating the Histopathological and Clinicoradiological Features Avoids a Diagnostic Pitfall. International journal of surgical pathology, 25(2), 141-147.

8. Stathopoulos, P., Mezitis, M., Kappatos, C., Titsinides, S., \& Stylogianni, E. (2011). Cysts and tumors associated with impacted third molars: is prophylactic removal justified?. Journal of oral and maxillofacial surgery, 69(2), 405-408.

9. da Silva, T. A., Batista, A. C., Mendonça, E. F., Leles, C. R., Fukada, S., \& Cunha, F. Q. (2008). Comparative expression of RANK, RANKL, and OPG in keratocystic odontogenic tumors, ameloblastomas, and dentigerous cysts. Oral surgery, oral medicine, oral pathology, oral radiology, and endodontology, 105(3), 333-341.

10. Bisinelli, J. C., Ioshii, S., Retamoso, L. B., Moysés, S. T., Moysés, S. J., \& Tanaka, O. M. (2010). Conservative treatment of unicystic ameloblastoma. American Journal of Orthodontics and Dentofacial Orthopedics, 137(3), 396-400.

11. Gendviliene, I., Legrand, P., Nicolielo, L. F. P., Sinha, D., Spaey, Y., Politis, C., \& Jacobs, R. (2017). Conservative management of large mandibular dentigerous cysts with a novel approach for follow up: Two case reports. Stomatologija, 19(1), 24-32. 